1 Hacettepe Journal of Mathematics and Statistics

$\bigcap$ Volume 45 (3) (2016), 765 - 780

\title{
Strongly copure projective objects in triangulated categories
}

\author{
Xin $\mathrm{Ma}^{*}$ and Zhongkui Liu
}

\begin{abstract}
In this paper, we introduce and investigate the notions of $\xi$-strongly copure projective objects in a triangulated category. This extends Asadollahi's notion of $\xi$-Gorenstein projective objects. Then we study the $\xi$-strongly copure projective dimension and investigate the existence of $\xi$-strongly copure projective precover.
\end{abstract}

Keywords: strongly copure projective object; triangulated category; proper class of triangles.

2000 AMS Classification: 18E30; 18G25; $18 \mathrm{G} 20$.

Received : 18.01.2015 Accepted : 23.06.2015 Doi : 10.15672/HJMS.20164513098

\section{Introduction}

Triangulated categories originated from algebraic geometry and algebraic topology and were introduced by Grothendieck and Verdier in the early sixties as the proper framework for doing homological algebra in an abelian category. By now triangulated categories have become indispensable in many different areas of mathematics, such as algebraic geometry, stable homotopy theory, and representation theory.

In [3], Beligiannis develops a classical homological algebra in a triangulated category $\mathcal{C}=$ $(\mathcal{C}, \Sigma, \Delta)$. He introduced $\xi$-projective objects, $\xi$-projective resolution, $\xi$-projective dimension and their dualities. Based on the works of Auslander and Bridger [2], Enochs and Jenda [8] and Beligiannis [5], Asadollahi [3] introduced and studied $\xi$-Gorenstein projective objects and their dualities, which maked contributions to develop there relative homological algebra in a triangulated category.

At the other extreme, Mao [9] investigated strongly $P$-projective modules. $M$ is called to be strongly $P$-projective if $\operatorname{Ext}_{R}^{i}(M, P)=0$ for all projective left $R$-modules $P$, which is dual to strongly copure injective modules in Enochs and Jenda [6]. So we also call strongly $P$-projective modules as strongly copure projective modules in this paper. As we all known, strongly copure projective (resp.

*Department of Mathematics, Northwest Normal University, Lanzhou 730070, China.

Email : maxin263@126.com

Science College, Gansu Agricultural University, Lanzhou 730070, China

Email : liuzkenwnu.edu.cn 
injective) modules are a generaliation of Gorenstein projective and projective (resp., Gorenstein injective and injective) modules in categories of modules.

Our aim in this paper is to introduce and study $\xi$-strongly copure projective (injective) objects in a triangulated category $\mathcal{C}$. This is denoted by $\xi$-SCprojective $(\xi$-SCinjective) objects for convenience. In Section 2, we introduce the notion of $\xi$-SCprojective objects and study some properties of $\xi$-SCprojective objects in $\mathcal{C}$. We also investigate $\xi$-SCprojective dimension. In Section 3, we introduce the concept of $\xi$-SCprojective precover and show the existence of $\xi$-SCprojective precover.

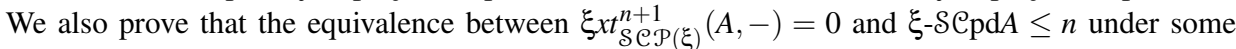
conditions.

Next we recall some known notions and facts of triangulated categories needed in the sequel. The basic reference for triangulated categories and derived categories is the original article of Verdier [15]. Also $[3,7,11]$ give introduction to these concepts.

Let $\mathcal{C}$ be an additive category and $\Sigma: \mathcal{C} \longrightarrow \mathcal{C}$ an additive functor. Let $\operatorname{Diag}(\mathcal{C}, \Sigma)$ denotes the category whose objects are diagrams in $\mathcal{C}$ of the form $A \longrightarrow B \longrightarrow C \longrightarrow \Sigma A$, and morphisms between two objects $A_{i} \longrightarrow B_{i} \longrightarrow C_{i} \longrightarrow \Sigma A_{i}, i=1,2$, are triple of morphisms $\alpha: A_{1} \longrightarrow A_{2}$, $\beta: B_{1} \longrightarrow B_{2}$ and $\gamma: C_{1} \longrightarrow C_{2}$, such that the following diagram commutes:

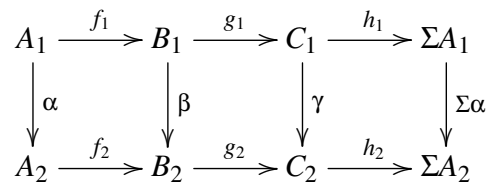

A triangle $(\mathcal{C}, \Sigma, \Delta)$ is called a triangulated category, where $\mathcal{C}$ is an additive category. $\Sigma$ is an autoequivalence of $\mathcal{C}$ and $\Delta$ is a full subcategory of $\operatorname{Diag}(\mathcal{C}, \Sigma)$ which satisfies the following axioms. The elements of $\Delta$ are then called triangles.

(Tr1) Every diagram isomorphic to a triangle is a triangle. Every morphism $f: A \longrightarrow B$ in $\mathcal{C}$ can be embedded into a triangle $A \stackrel{f}{\longrightarrow} B \stackrel{g}{\longrightarrow} C \stackrel{h}{\longrightarrow} \Sigma A$. For any object $A \in \mathcal{C}$, the diagram $0 \longrightarrow A \stackrel{1_{A}}{\longrightarrow} A \longrightarrow 0$ is a triangle, where $1_{A}$ denotes the identity morphism from $A$ to $A$.

(Tr2) $A \stackrel{f}{\longrightarrow} B \stackrel{g}{\longrightarrow} C \stackrel{h}{\longrightarrow} \Sigma A$ is a triangle if and only if $B \stackrel{g}{\longrightarrow} C \stackrel{h}{\longrightarrow} \Sigma A \stackrel{-\Sigma f}{\longrightarrow} \Sigma B$ is so.

(Tr3) Given triangles $A_{i} \stackrel{f_{i}}{\longrightarrow} B_{i} \stackrel{g_{i}}{\longrightarrow} C_{i} \stackrel{h_{i}}{\longrightarrow} \Sigma A_{i}, i=1,2$, and morphisms $\alpha: A_{1} \longrightarrow A_{2}$ and $\beta: B_{1} \longrightarrow B_{2}$ such that $\alpha f_{2}=f_{1} \beta$, there exists a morphism $\gamma: C_{1} \longrightarrow C_{2}$ such that $(\alpha, \beta, \gamma)$ is a morphism from the first triangle to the second.

(Tr4) (The Octahedral Axiom) Given triangles $A \stackrel{f}{\longrightarrow} B \stackrel{i}{\longrightarrow} C^{\prime} \stackrel{i^{\prime}}{\longrightarrow} \Sigma A, B \stackrel{g}{\longrightarrow} C \stackrel{j}{\longrightarrow} A^{\prime} \stackrel{f^{\prime}}{\longrightarrow} \Sigma B$, $A \stackrel{g f}{\longrightarrow} C \stackrel{k}{\longrightarrow} B^{\prime} \stackrel{k^{\prime}}{\longrightarrow} \Sigma A$, there exist morphisms $f^{\prime}: C^{\prime} \longrightarrow B^{\prime}$ and $g^{\prime}: B^{\prime} \longrightarrow A^{\prime}$ such that the following diagram commutes and the third row is triangle:

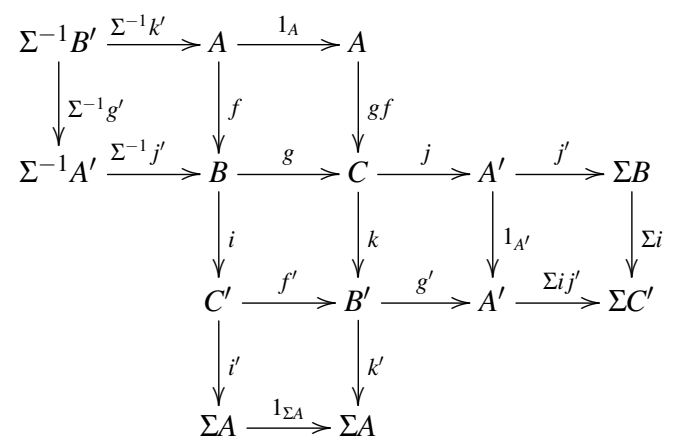

Throughout the paper, we fix a triangulated category $\mathcal{C}=(\mathcal{C}, \Sigma, \Delta), \Sigma$ is the suspension functor and $\Delta$ is the triangulation. 
1.1. Proposition. ([3, Proposition 2.1]) Let $\mathcal{C}$ be an additive category equipped with an autoequivalence $\Sigma: \mathcal{C} \longrightarrow \mathcal{C}$ and a class of diagrams $\Delta \subseteq \operatorname{Diag}(\mathcal{C}, \Sigma)$. Suppose that the triple $(\mathcal{C}, \Sigma, \Delta), \Sigma$ satisfies all the axioms of a triangulated category except possibly of the Octahedral Axiom. Then the following are equivalent:

(a) Base change. For any triangle $A \stackrel{f}{\longrightarrow} B \stackrel{g}{\longrightarrow} C \stackrel{h}{\longrightarrow} \Sigma A \in \Delta$ and morphism $\varepsilon: E \longrightarrow C$, there exists a commutative diagram

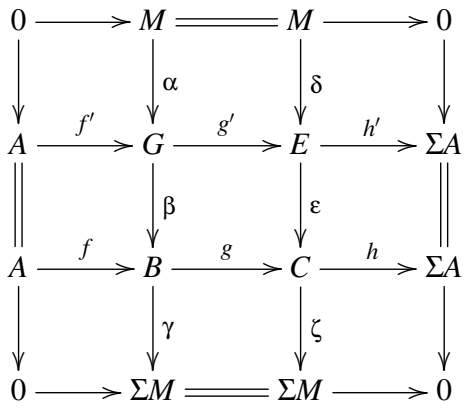

in which all horizontal and vertical diagrams are triangle in $\Delta$.

(b) Cobase change. For any triangle $A \stackrel{f}{\longrightarrow} B \stackrel{g}{\longrightarrow} C \stackrel{h}{\longrightarrow} \Sigma A \in \Delta$ and any morphism $\alpha: A \longrightarrow D$, there exists a commutative diagram

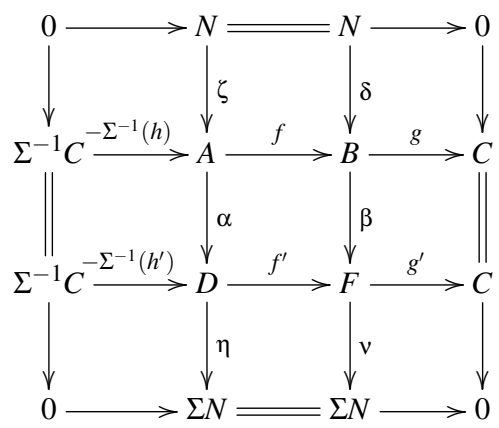

in which all horizontal and vertical diagrams are triangles in $\Delta$.

(c) Octahedral Axiom For any two morphisms $f_{1}: A \longrightarrow B, f_{2}: B \longrightarrow C$, there exists a commutative diagram

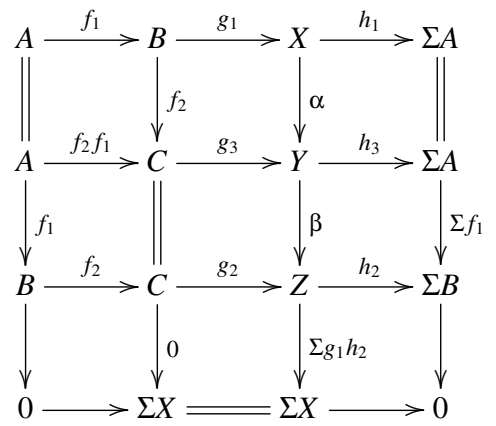

in which all horizontal and the third vertical diagrams are triangles in $\Delta$.

A class of triangles $\xi$ is closed under base change if for any triangle $A \stackrel{f}{\longrightarrow} B \stackrel{g}{\longrightarrow} C \stackrel{h}{\longrightarrow} \Sigma A \in \xi$ and any morphism $\varepsilon: E \longrightarrow C$ as in Proposition 1.1(a), the triangle $A \stackrel{f^{\prime}}{\longrightarrow} G \stackrel{g^{\prime}}{\longrightarrow} E \stackrel{h^{\prime}}{\longrightarrow} \Sigma A$ belongs 
to $\xi$. Dually, a class of triangles is closed under cobase change if for any triangle $A \stackrel{f}{\longrightarrow} B \stackrel{g}{\longrightarrow} C \stackrel{h}{\longrightarrow}$ $\Sigma A \in \xi$ and any morphism $\alpha: A \longrightarrow D$ as in Proposition 1.1(b), the triangle $D \stackrel{f^{\prime}}{\longrightarrow} F \stackrel{g^{\prime}}{\longrightarrow} C \stackrel{h^{\prime}}{\longrightarrow} \Sigma D$ belongs to $\xi$. A class of triangles is closed under suspension if for any triangle $A \stackrel{f}{\longrightarrow} B \stackrel{g}{\longrightarrow} C \stackrel{h}{\longrightarrow}$ $\Sigma A \in \xi$ and any integer $i \in \mathbb{Z}$, the triangle

$$
\Sigma^{i} A \stackrel{(-1)^{i} \Sigma^{i} f}{\longrightarrow} \Sigma^{i} B \stackrel{(-1)^{i} \Sigma^{i} g}{\longrightarrow} \Sigma^{i} C \stackrel{(-1)^{i} \Sigma^{i} h}{\longrightarrow} \Sigma^{i+1} A
$$

is in $\xi$. A class of triangles $\xi$ is called saturated if in the situation of base change in Proposition 1.1, whenever the third vertical and the second horizontal triangle is in $\xi$, then the triangle $A \stackrel{f}{\longrightarrow} B \stackrel{g}{\longrightarrow}$ $C \stackrel{h}{\longrightarrow} \Sigma A$ is in $\xi$.

1.2. Definition. ([3, Definition 2.2]) A full subcategory $\xi \subseteq \operatorname{Diag}(\mathcal{C}, \Sigma)$ is called a proper class of triangles if the following conditions hold:

(i) $\xi$ is closed under isomorphisms, finite coproducts and $\Delta_{0} \subseteq \xi \subseteq \Delta$, where $\Delta_{0}$ denotes the full subcategory of split triangles.

(ii) $\xi$ is closed under suspensions and is saturated.

(iii) $\xi$ is closed under base and cobase change.

Throughout we fix a proper class of triangles $\xi$ in the triangulated category $\mathcal{C}$.

\section{Strongly copure projective objects}

2.1. Definition. ([3, Definition 4.1]) An object $P \in \mathcal{C}$, (respectively $I \in \mathcal{C})$ is called $\xi$-projective (respectively $\xi$-injective) if for any triangle $A \rightarrow B \rightarrow C \rightarrow \Sigma A$ in $\xi$, the induced sequence

$$
\begin{gathered}
0 \rightarrow \operatorname{Hom}_{\mathcal{C}}(P, A) \rightarrow \operatorname{Hom}_{\mathcal{C}}(P, B) \rightarrow \operatorname{Hom}_{\mathcal{C}}(P, C) \rightarrow 0 \\
\text { (respectively } \left.0 \rightarrow \operatorname{Hom}_{\mathcal{C}}(C, I) \rightarrow \operatorname{Hom}_{\mathcal{C}}(B, I) \rightarrow \operatorname{Hom}_{\mathcal{C}}(A, I) \rightarrow 0\right)
\end{gathered}
$$

is exact in the category of abelian group $\mathcal{A b}$.

The symbol $\mathcal{P}(\xi)$ (res. $\mathcal{J}(\xi)$ ) will denote the full subcategory of $\xi$-projective (res. $\xi$-injective) objects of $\mathcal{C}$. It follows easily from the definition that the categories $\mathcal{P}(\xi)$ and $\mathcal{J}(\xi)$ are full, additive, closed under isomorphisms, direct summands and $\Sigma$-stable.

$\mathcal{C}$ is said to have enough $\xi$-projective objects if for any object $A \in \mathcal{C}$ there exists a triangle $K \rightarrow P \rightarrow A \rightarrow \Sigma K$ in $\xi$ with $P \in \mathcal{P}(\xi)$. Dually one defines when $\mathcal{C}$ has enough $\xi$-injectives.

2.2. Lemma. ([3, Lemma 4.2]) Assume that $\mathcal{C}$ is a triangulated category with enough $\xi$-projective objects. Then $A \longrightarrow B \longrightarrow C \longrightarrow \Sigma A$ is in $\xi$ if and only if for all $P \in \mathcal{P}(\xi)$ the induced sequence $0 \longrightarrow \operatorname{Hom}_{\mathcal{C}}(P, A) \longrightarrow \operatorname{Hom}_{\mathcal{C}}(P, B) \longrightarrow \operatorname{Hom}_{\mathcal{C}}(P, C) \longrightarrow 0$ is exact.

In [3], the $\xi$-projective dimension $\xi$-pd $A$ of an object $A \in \mathcal{C}$ is defined inductively.

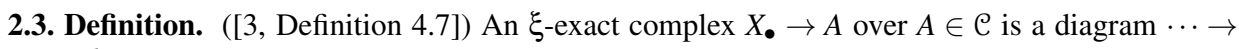
$X_{n+1} \stackrel{d_{n+1}}{\longrightarrow} X_{n} \longrightarrow \cdots \longrightarrow X_{1} \stackrel{d_{1}}{\longrightarrow} X_{0} \stackrel{d_{0}}{\longrightarrow} A \rightarrow 0$ such that for each integer $n \geq 0$ :

(i) There are triangles $K_{n+1} \stackrel{g_{n}}{\longrightarrow} X_{n} \stackrel{f_{n}}{\longrightarrow} K_{n} \stackrel{h_{n}}{\longrightarrow} \Sigma K_{n+1}$ in $\xi$, where $K_{0}=A$.

(ii) The differential $d_{n}=g_{n-1} f_{n}$ for any $n \geq 1$ and $d_{0}=f_{0}$.

An $\xi$-projective resolution of $A \in \mathcal{C}$ is an $\xi$-exact complex $P_{\bullet} \rightarrow A$ as above such that $P_{n} \in \mathcal{P}(\xi)$, $n \geq 0$.

2.4. Definition. ([2, Definition, 3.2]) A triangle $A \rightarrow B \rightarrow C \rightarrow \Sigma A$ in $\xi$ is called $\operatorname{Hom}_{\mathcal{C}}(-, \mathcal{P}(\xi))$ exact, if for any $Q \in \mathcal{P}(\xi)$, the induced complex

$$
0 \rightarrow \operatorname{Hom}_{\mathcal{C}}(C, Q) \rightarrow \operatorname{Hom}_{\mathcal{C}}(B, Q) \rightarrow \operatorname{Hom}_{\mathcal{C}}(A, Q) \rightarrow 0
$$

is exact in $\mathcal{A b}$. 
2.5. Definition. An object $A$ is said to be $\xi$-strongly copure projective object ( $\xi$-SCprojective object) if there exists an $\xi$-projective resolution of $A: \cdots \rightarrow P_{n+1} \stackrel{d_{n+1}}{\longrightarrow} P_{n} \rightarrow \cdots \rightarrow P_{1} \stackrel{d_{0}}{\rightarrow} P_{0} \stackrel{d_{0}}{\rightarrow} A \rightarrow 0$ with $P_{i} \in \mathcal{P}(\xi)$ for all $i \geq 0$ such that $K_{n+1} \stackrel{g_{n}}{\rightarrow} P_{n} \stackrel{f_{n}}{\rightarrow} K_{n} \stackrel{h_{n}}{\longrightarrow} \Sigma K_{n+1}$ in $\xi$ are $\operatorname{Hom}_{\mathcal{C}}(-, \mathcal{P}(\xi))$-exact triangles for all integer $n$.

We denote $\operatorname{SeP}(\xi)$ the full subcategory of $\xi$-strongly copure projective objects of $\mathcal{C}$. It follows directly from the definition that the category $\operatorname{SeP}(\xi)$ is full, additive and closed under isomorphisms.

Remark. By [2, Definition 3.6], every $\xi$-Gorenstein projective object is $\xi$-strongly copure projective. In particular, there is an inclusion of categories $\mathcal{G P}(\xi) \subseteq \operatorname{SeP}(\xi)$, where $\mathcal{G P}(\xi)$ is the class of $\xi$-Gorenstein projective objects.

Let $C$ be an object of $\mathcal{C}$. For any integer $n \geq 0$, the $\xi$-extension functor $\xi x t_{\xi}^{n}(-, C)$ is defined to be the $n$th right $\xi$-derived functor of the functor $\operatorname{Hom}_{\mathcal{C}}(-, C)$, that is $\xi_{x} t_{\xi}^{n}(-, C):=R_{\xi}^{n} \operatorname{Hom}_{\mathcal{C}}(-, C)$.

2.6. Proposition. ([3, Corollary 4.12]) If $A \longrightarrow B \longrightarrow C \longrightarrow \Sigma A$ is a triangle in $\xi$, then for any $X \in \mathcal{C}$ we have a long exact sequence

$$
0 \longrightarrow \xi x t_{\xi}^{0}(C, X) \longrightarrow \xi x t_{\xi}^{0}(B, X) \longrightarrow \xi x t_{\xi}^{0}(A, X) \longrightarrow \xi x t_{\xi}^{1}(C, X) \longrightarrow \cdots
$$

2.7. Lemma. Let $A$ be a $\xi$-SCprojective object of $\mathcal{C}$. Then $\xi_{x t}(A, Q) \cong \operatorname{Hom}_{\mathcal{C}}(A, Q)$ and $\xi x t_{\xi}^{i}(A, Q)=0$ for any $Q \in \widetilde{\mathcal{P}}(\xi)$ and any $i>0$, where $\widetilde{\mathcal{P}}(\xi)$ denote the full subcategory of $\mathcal{C}$ whose objects are of finite $\xi$-projective dimension.

Proof. Let $\xi$-pd $Q=n$ for some nonnegative integer $n$ and $P_{*}$ a $\xi$-projective resolution of $A$. If $n=0$, then $Q$ is an $\xi$-projective object. Then $\operatorname{Hom}_{\mathcal{C}}\left(P_{*}, Q\right)$ is an exact sequence, and this implies that

$$
\operatorname{Hom}_{\mathcal{C}}(A, Q) \cong H^{0}\left(0 \longrightarrow \operatorname{Hom}_{\mathcal{C}}\left(P_{0}, Q\right) \longrightarrow \operatorname{Hom}_{\mathcal{C}}\left(P_{1}, Q\right) \longrightarrow \cdots\right) \cong \xi x t_{\xi}^{0}(A, Q) .
$$

Moreover, $\xi x t_{\xi}^{i}(A, Q)=0$ for any $i>0$. Inductively, suppose that the assertions follow for any object with $\xi$-projective dimension $n-1$. Consider the triangle $K \longrightarrow P \longrightarrow Q \longrightarrow \Sigma K$ in $\xi$, where $P \in$ $\mathcal{P}(\xi)$ and $\xi$-pd $K=n-1$. For any $j \in \mathbb{Z}$, the triangle $\Sigma^{j} K \stackrel{(-1)^{j} \Sigma^{j} f}{\longrightarrow} \Sigma^{j} P \stackrel{(-1)^{j} \Sigma^{j} g}{\longrightarrow} \Sigma^{j} Q \stackrel{(-1)^{j} \Sigma^{j} h}{\longrightarrow} \Sigma^{j+1} K$ is also in $\xi$. By Proposition 2.6, there is an exact sequence $0 \longrightarrow \xi x t_{\xi}^{0}\left(A, \Sigma^{j} K\right) \longrightarrow \xi x t_{\xi}^{0}\left(A, \Sigma^{j} P\right)$, and then $0 \longrightarrow \operatorname{Hom}_{\mathcal{C}}\left(A, \Sigma^{j} K\right) \longrightarrow \operatorname{Hom}_{\mathcal{C}}\left(A, \Sigma^{j} P\right)$. This implies that $\operatorname{Hom}_{\mathcal{C}}(A,-)$ kills $\xi$-phantom map $(-1)^{j} \Sigma^{j} h$. Especially, we have the following commutative diagram:

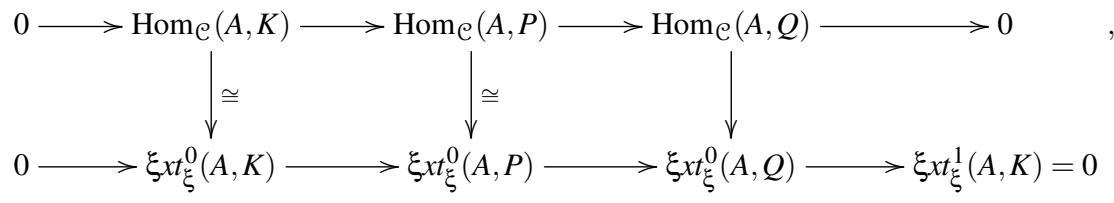

where rows are exact. Hence $\xi x t_{\xi}^{0}(A, Q) \cong \operatorname{Hom}_{\mathcal{C}}(A, Q)$. Since

$$
\xi x t_{\xi}^{i}(A, P) \longrightarrow \xi x t_{\xi}^{i}(A, Q) \longrightarrow \xi x t_{\xi}^{i+1}(A, K)
$$

is exact by Proposition 2.6, where $\xi x t_{\xi}^{i}(A, P)=\xi x t_{\xi}^{i+1}(A, K)=0$. Thus $\xi x t_{\xi}^{i}(A, Q)=0$.

2.8. Proposition. Assume that $\mathcal{C}$ is a triangulated category with enough $\xi$-projective objects and $X$ is an object in $\mathcal{P}(\xi)$. Then $X$ is $\xi$-injective relative to $\operatorname{SeP}(\xi)$.

Proof. Let $A \longrightarrow B \longrightarrow C \longrightarrow \Sigma A$ be a triangle of $\mathcal{S C P}(\xi)$ in $\xi$. By Proposition 2.6, there is an exact sequence $0 \longrightarrow \xi x t_{\xi}^{0}(C, X) \longrightarrow \xi_{x} t_{\xi}^{0}(B, X) \longrightarrow \xi_{\xi} t_{\xi}^{0}(A, X) \longrightarrow \xi x t_{\xi}^{1}(C, X)$. Since $\xi x t_{\xi}^{1}(C, X)=$ 0 by Lemma 2.7 and $\xi x t_{\xi}^{0}(G, X) \cong \operatorname{Hom}_{\mathcal{C}}(G, X)$ for any $G \in \mathcal{S} \mathcal{P P}(\xi)$, there is an exact sequence $0 \longrightarrow \operatorname{Hom}_{\mathcal{C}}(C, X) \longrightarrow \operatorname{Hom}_{\mathcal{C}}(B, X) \longrightarrow \operatorname{Hom}_{\mathcal{C}}(A, X) \longrightarrow 0$. So $X$ is $\xi$-injective relative to $\mathcal{S} \mathcal{C P}(\xi)$. 
2.9. Theorem. Assume that $\mathcal{C}$ is a triangulated category with enough $\xi$-projective objects and $A \stackrel{\alpha}{\longrightarrow}$ $B \stackrel{\beta}{\longrightarrow} C \stackrel{\gamma}{\longrightarrow} \Sigma A$ is a triangle in $\xi$ such that $C$ is $\xi$-SCprojective. Then $A$ is $\xi$-SCprojective if and only if $B$ is $\xi$-SCprojective.

Proof. First assume that $A$ is $\xi$-SCprojective. We will show that $B$ is also such. Since $A$ and $C$ are $\xi$-SCprojective, there exist triangles $K_{A} \stackrel{g_{A}}{\longrightarrow} P_{A} \stackrel{f_{A}}{\longrightarrow} A \stackrel{h_{A}}{\longrightarrow} \Sigma K_{A}$ and $K_{C} \stackrel{g_{C}}{\longrightarrow} P_{C} \stackrel{f_{C}}{\longrightarrow} C \stackrel{h_{C}}{\longrightarrow} \Sigma K_{C}$ in $\xi$, where $P_{A}$ and $P_{C}$ are $\xi$-projective, $K_{A}$ and $K_{C}$ are $\xi$-SCprojective. By [3, Lemma 4.2], $\gamma f_{C}=0$. Using that $\Sigma$ is an automorphism and a result of Verdier [16], the commutative square on the top left corner below is embedded in a diagram

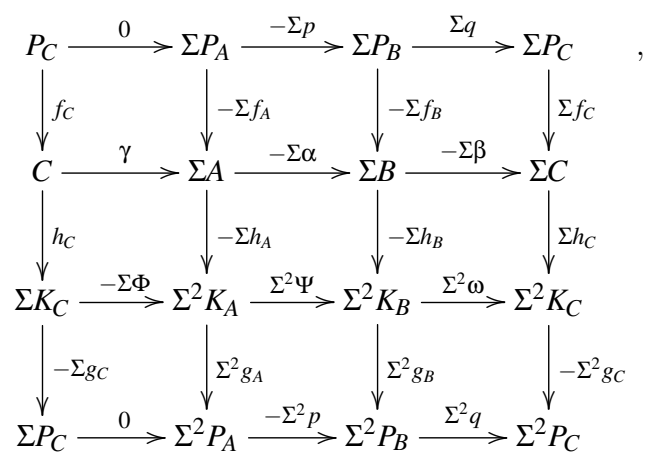

which is commutative except the lower right square which anticommutes and where the rows and columns are triangles. But the above diagram is equivalent to the following commutative diagram:

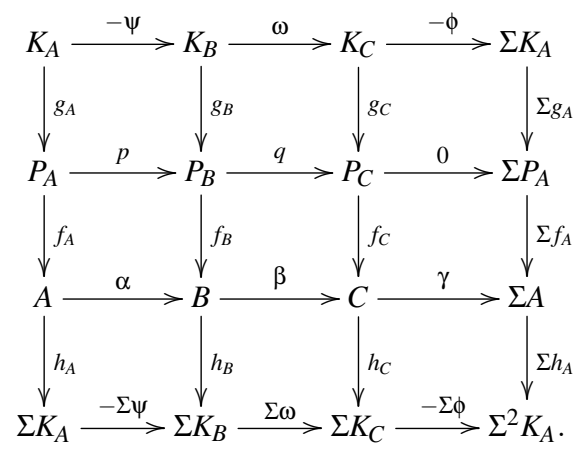

Since the second horizontal triangle is split and $P_{A}, P_{C}$ are $\xi$-projective, $P_{B}$ is $\xi$-projective. Applying to the above diagram the homological functor $\operatorname{Hom}_{\mathcal{C}}(P,-), \forall P \in \mathcal{P}(\xi)$, a simple diagram chasing argument shows that $0 \longrightarrow \operatorname{Hom}_{\mathcal{C}}\left(P, K_{A}^{1}\right) \longrightarrow \operatorname{Hom}_{\mathcal{C}}\left(P, K_{B}^{1}\right) \longrightarrow \operatorname{Hom}_{\mathcal{C}}\left(P, K_{C}^{1}\right) \longrightarrow 0$ is exact. By Lemma 2.2, the first horizontal triangle is in $\xi$. Similarly the sencond vertical triangle is in $\xi$. Since there is the commutative diagram for any $Q \in \mathcal{P}(\xi)$ : 


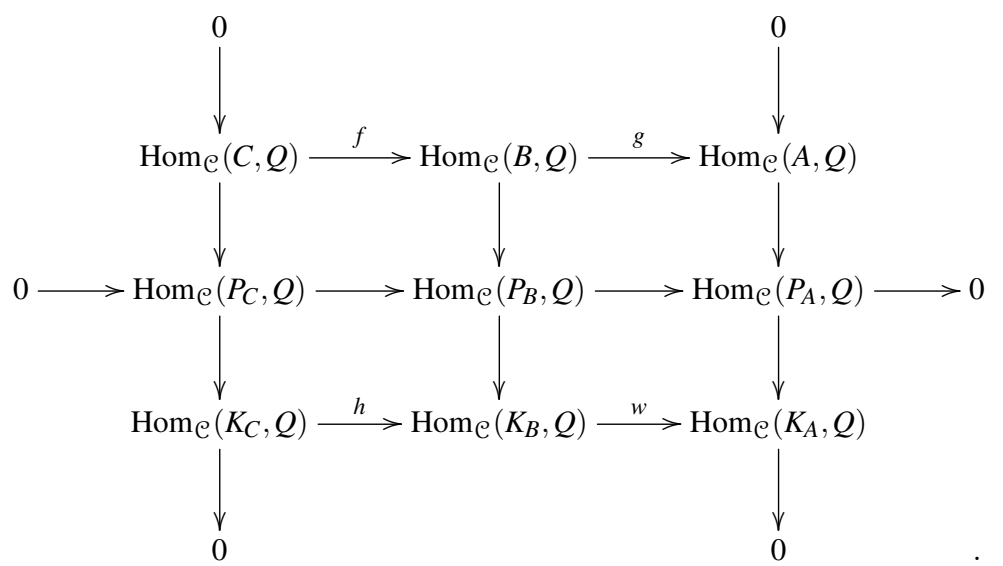

Obviously, $f$ is monic and $w$ is epic. Thus $g$ is epic and $h$ is monic. By $\operatorname{Hom}_{\mathcal{C}}(-, Q)$ is a cohomological functor and snake Lemma, the sequence $0 \longrightarrow \operatorname{Hom}_{\mathcal{C}}(B, Q) \longrightarrow \operatorname{Hom}_{\mathcal{C}}\left(P_{B}, Q\right) \longrightarrow$ $\operatorname{Hom}_{\mathcal{C}}\left(K_{B}, Q\right) \longrightarrow 0$ is exact. Proceeding the above procedure for the triangle $K_{A} \longrightarrow K_{B} \longrightarrow$ $K_{C} \longrightarrow \Sigma K_{A}$, we get the $\xi$-projective resolution of $B$ with appropriate properties. Hence $B$ is $\xi$ SCprojective.

Assume that $B$ is $\xi$-SCprojective. By base change, there is a commutative diagram:

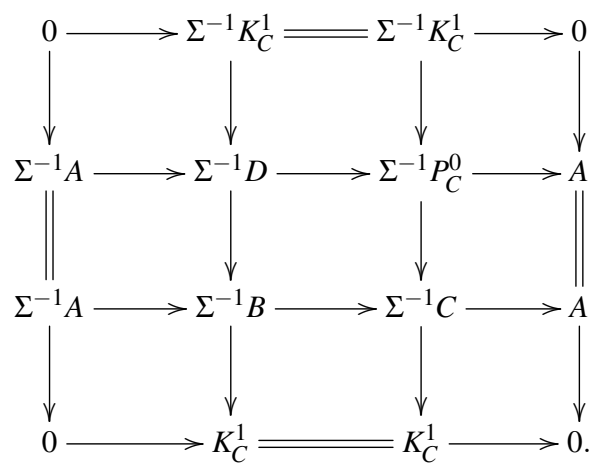

Since $\Sigma^{-1} B$ and $\Sigma^{-1} K_{C}^{1}$ are $\xi$-SCprojective, we may use the previous case to deduce that $\Sigma^{-1} D$ is $\xi$ SCprojective. Then there exists an $\xi$-projective resolution of $\Sigma^{-1} D: \cdots \longrightarrow \Sigma^{-1} P_{D}^{1} \longrightarrow \Sigma^{-1} P_{D}^{0} \longrightarrow$ $\Sigma^{-1} D$ satisfying the condition of definition. Since $C$ is $\xi$-SCprojective, there exists a triangle $K_{C}^{1} \stackrel{g_{C}}{\longrightarrow}$ $P_{C}^{0} \stackrel{f_{C}}{\longrightarrow} C \stackrel{h_{C}}{\longrightarrow} \Sigma K_{C}$ in $\xi$ with $P_{C}^{0} \xi$-projective and $K_{C}^{1} \xi$-SCprojective and $K_{C}^{1} \longrightarrow P_{C}^{0} \longrightarrow C \longrightarrow \Sigma K_{C}^{1}$ is $\operatorname{Hom}_{\mathcal{C}}(-, \mathcal{P}(\xi))$ exact. For any $Q \in \mathcal{P}(\xi)$,there is a commutative diagram:

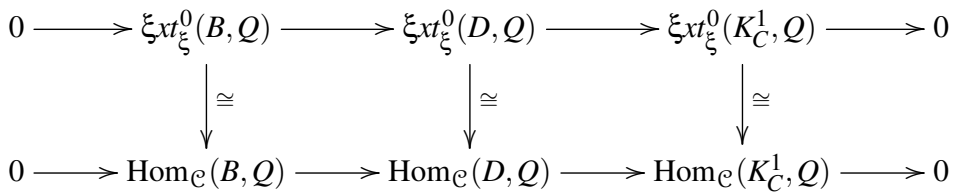


by Lemma 2.7. Moreover, there is the commutative diagram

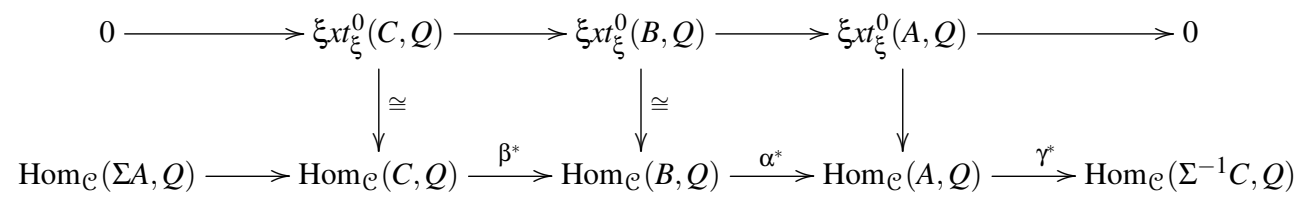

with the below is exact. Since $\beta^{*}$ is monic, $\gamma^{*}$ is also so. So $0 \longrightarrow \operatorname{Hom}_{\mathcal{C}}(C, Q) \longrightarrow$ $\operatorname{Hom}_{\mathcal{C}}(B, Q) \longrightarrow \operatorname{Hom}_{\mathcal{C}}(A, Q) \longrightarrow 0$ is exact. Thus $\Sigma^{-1} A \longrightarrow \Sigma^{-1} D \longrightarrow \Sigma^{-1} P_{C}^{0} \longrightarrow A$ is $\operatorname{Hom}_{\mathcal{C}}(-, \mathcal{P}(\xi))$ exact. Now pasting $\cdots \longrightarrow \Sigma^{-1} P_{D}^{1} \longrightarrow \Sigma^{-1} P_{D}^{0} \longrightarrow \Sigma^{-1} D$ with $\Sigma^{-1} A \longrightarrow$ $\Sigma^{-1} D \longrightarrow \Sigma^{-1} P_{C}^{0} \longrightarrow A$, so $A$ is $\xi$-SCprojective.

2.10. Proposition. Assume that $\mathcal{C}$ is a triangulated category with enough $\xi$-projective objects. If $X \in \operatorname{SeP}(\xi)$ is $\xi$-projective relative to $\mathcal{S} \mathcal{P}(\xi)$, then $X \in \mathcal{P}(\xi)$.

Proof. Since $\mathcal{C}$ has enough $\xi$-projectives, there exists a triangle $K \longrightarrow P \longrightarrow X \longrightarrow \Sigma K$ in $\xi$ with $P \in \mathcal{P}(\xi)$. But $X$ and $P$ are $\xi$-SCprojective, then so is $K$ by Theorem 2.9. Since $X$ is $\xi$-projective relative to $\operatorname{SeP}(\xi)$, there exists an exact sequence

$$
0 \longrightarrow \operatorname{Hom}_{\mathcal{C}}(X, K) \longrightarrow \operatorname{Hom}_{\mathcal{C}}(X, P) \longrightarrow \operatorname{Hom}_{\mathcal{C}}(X, X) \longrightarrow 0 .
$$

So $K \longrightarrow P \longrightarrow X \longrightarrow \Sigma K$ is split. Then $P \cong K \oplus X$. Hence $X \in \mathcal{P}(\xi)$.

It is clear that $\operatorname{SeP}(\xi)$ is closed under countable direct sums. In the following, we use Eilenberg's trick to show that $\mathcal{S} \mathcal{P} \mathcal{P}(\xi)$ is closed under direct summands.

2.11. Corollary. $\operatorname{SeP}(\xi)$ is closed under direct summands.

Proof. Let $A$ be an object of $\operatorname{SeP}(\xi)$ and $B$ a direct summand of $A$. So $A=B \oplus B^{\prime}$, for some object $B^{\prime}$ of $\mathcal{C}$. Set

$$
K=B \oplus B^{\prime} \oplus B \oplus B^{\prime} \oplus \cdots .
$$

Since $K=A \oplus A \oplus \cdots$ and $\mathcal{S} \mathcal{P}(\xi)$ is closed under countable direct sum, $K$ belongs to $\mathcal{S} \mathcal{P}(\xi)$. We have $K \cong B \oplus K$ and so $B \oplus K$ also belongs to $\mathcal{S C P}(\xi)$. Now consider the split exact triangle

$$
B \longrightarrow B \oplus K \longrightarrow K \stackrel{0}{\longrightarrow} \Sigma B
$$

in $\xi$ to conclude, from the previous Theorem 2.9 , that $B$ belongs to $\operatorname{SeP}(\xi)$.

Now we introduce a new invariant for an object $A$ of $\mathcal{C}$, namely its $\xi$-SCprojective dimension, $\xi$ $\mathcal{S} \operatorname{epd} A$. It is defined inductively. When $\mathrm{A}=0$, put $\xi-\mathcal{S} \operatorname{Cpd} A=-1$. If $A \in \operatorname{SCP}(\xi)$, then $\xi-\mathcal{S} \operatorname{Cpd} A=0$. Next by induction, for an integer $n>0$, put $\xi-\mathcal{S C p d} A \leq n$ if there exists a triangle $K \longrightarrow P \longrightarrow A \longrightarrow$ $\Sigma K$ in $\mathcal{C}$ with $P \in \operatorname{SeP}(\xi)$ and $\xi-\mathcal{S} \mathcal{C p d} K \leq n-1$.

We define $\xi$-Sepd $A=n$ if $\xi$-Sepd $A \leq n$ and $\xi$-Sepd $A \not \leq n-1$. If $\xi$-SCpd $A \neq n$ for all $n \geq 0$, we set $\xi-\mathcal{S} \operatorname{epd} A=\infty$.

2.12. Theorem. Assume that $\mathcal{C}$ is a triangulated category with enough $\xi$-projective objects and $A \longrightarrow B \longrightarrow C \stackrel{\gamma}{\longrightarrow} \Sigma A$ is a triangle in $\xi$ such that $A \neq 0$ and $C$ is $\xi$-SCprojective. Then $\xi-\mathcal{S} \operatorname{Cpd} A=\xi$ $\operatorname{SCpd} B$.

Proof. The result is clear from Theorem 2.9 if one of $A$ or $B$ is $\xi$-SCprojective. Let $\xi$-SCpd $A=$ $n>0$. So there exists a triangle $K_{A} \longrightarrow P_{A} \longrightarrow A \longrightarrow \Sigma K_{A}$ in $\xi$ where $P_{A}$ is $\xi$-SCprojective and $\xi$-SCpd $K_{A}=n-1$. Since $C$ is $\xi$-SCprojective, there exists a triangle $K_{C} \longrightarrow P_{C} \longrightarrow C \longrightarrow \Sigma K_{C}$ in 
$\xi$ where $P_{C}$ is $\xi$-projective and $K_{C}$ is $\xi$-SCprojective. Then by the proof of Theorem 2.9, we have the following commutative diagram:

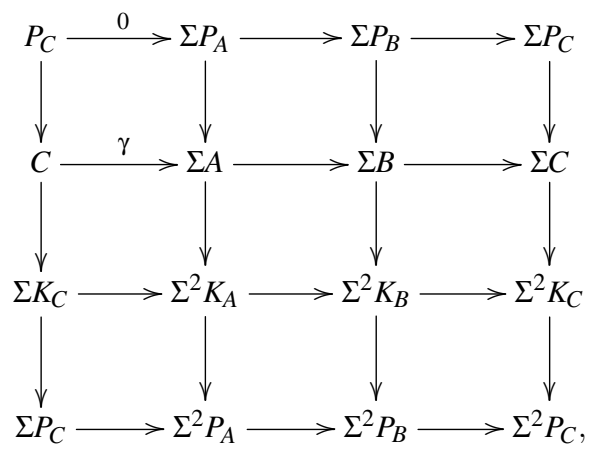

which is commutative except the lower right square which anticommutes and where the rows and columns are triangles. This is equivalent to the commutative diagram:

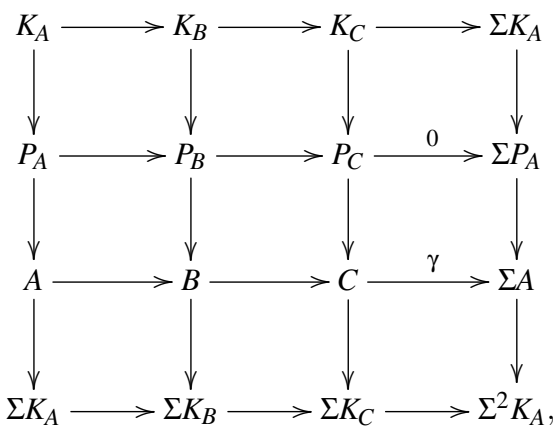

in which the first three vertical and horizontal diagrams are triangles. The second horizontal triangle is split, and so belongs to $\xi$. Since $P_{A}$ and $P_{C}$ are both $\xi$-SCprojective, it follows from that $P_{B}$ is also $\xi$-SCprojective. Applying $\operatorname{Hom}_{\mathcal{C}}(\mathcal{P}(\xi),-)$ to the above commutative diagram , by Lemma 2.2 and diagram chasing argument, the first horizontal and also second vertical triangles are $\operatorname{Hom}_{\mathcal{C}}(\mathcal{P}(\xi),-)$ exact and so belong to $\xi$. Now consider the triangle $K_{A} \longrightarrow K_{B} \longrightarrow K_{C} \longrightarrow \Sigma K_{A}$ in $\xi$, in which $\xi$ $\mathcal{S} \mathcal{C p d} K_{A}=n-1$ and use induction to deduce that $\xi-\mathcal{S} \operatorname{Cpd} K_{B}=n-1$ and hence $\xi-\mathcal{S} \operatorname{Cpd} B=n$.

Now suppose $\xi-\mathcal{S C} \mathrm{pd} B=n$. So there exists a triangle $K_{B} \longrightarrow P_{B} \longrightarrow B \longrightarrow \Sigma K_{B}$ in $\xi$, where $P_{B}$ is $\xi$-SCprojective and $\xi-\mathcal{S} \mathcal{C p d} K_{B}=n-1$. Using $(\operatorname{Tr} 2)$ and base change in Proposition 1.1, we get the following commutative diagram:

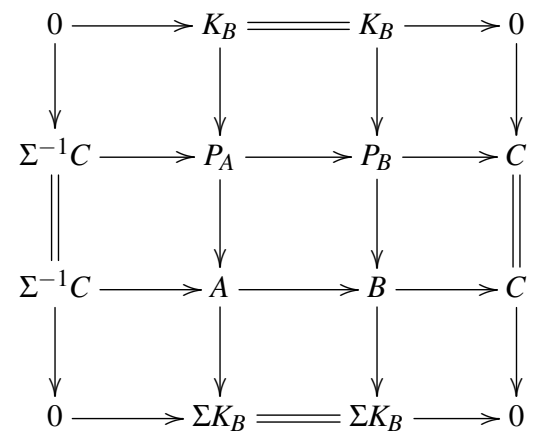


in which all horizontal and vertical diagrams are triangles. Since the third horizontal and third vertical triangles are in $\xi$, one can show the second horizontal and second vertical triangles are $\operatorname{Hom}_{\mathcal{C}}(\mathcal{P}(\xi),-)$ exact and so belong to $\xi$. Because both $P_{B}$ and $\Sigma^{-1} C$ are $\xi$-SCprojective, by Theorem 2.9, so is $P_{A}$. So $\xi-\mathcal{S} \operatorname{Cpd} A=n$.

2.13. Lemma. ([2, Proposition 3.15]) Let the following be a commutative diagram such that rows are triangles in $\xi$ :

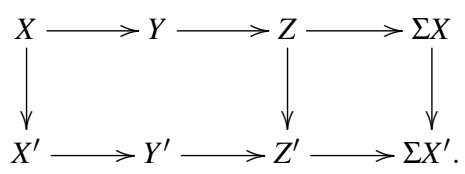

Then it may be completed to a morphism of triangles

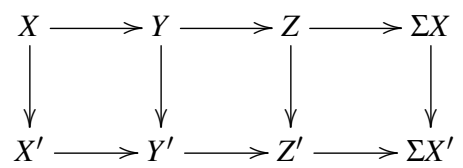

so that $X \longrightarrow X^{\prime} \oplus Y \longrightarrow Y^{\prime} \longrightarrow \Sigma X$ is a triangle in $\xi$.

2.14. Proposition. Assume that $\mathcal{C}$ is a triangulated category with enough $\xi$-projective objects and let $A$ be an object of $\mathcal{C}$. Then the following are equivalent:

(i) $\xi-\mathcal{S} \operatorname{epd} A \leq n$.

(ii) In any $\xi$-exact sequence $0 \longrightarrow B \longrightarrow P_{n-1} \longrightarrow \cdots \longrightarrow P_{0} \longrightarrow A \longrightarrow 0$, if $P_{i}$ are $\xi$ SCprojective, then so is $B$.

Proof. (i) $\Rightarrow$ (ii). There exits a triangle $K \longrightarrow Q \longrightarrow A \longrightarrow \Sigma K$ in $\xi$ where $Q$ is $\xi$-SCprojective and $\xi$-SCpd $K \leq n-1$. Since $0 \longrightarrow B \longrightarrow P_{n-1} \longrightarrow \cdots \longrightarrow P_{0} \longrightarrow A \longrightarrow 0$ is $\xi$-exact, by definition of $\xi$-exact sequence, there exists a triangle $K_{1} \longrightarrow P_{0} \longrightarrow A \longrightarrow \Sigma K$ in $\xi$. Since $\mathcal{C}$ have enough $\xi$-projectives, there exists a triangle $L \longrightarrow P \longrightarrow A \longrightarrow \Sigma L$ in $\xi$ with $P \xi$-projective. So we can construct morphisms of triangles:

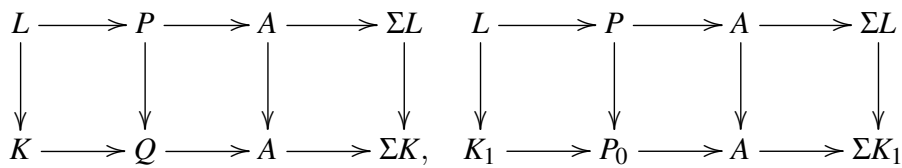

Now consider the diagrams

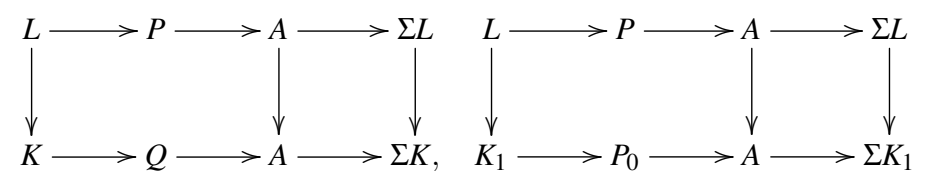

where the rows are triangles in $\xi$. By Lemma 2.13, we can complete them such that $L \longrightarrow K \oplus P \longrightarrow$ $Q \longrightarrow \Sigma L$ and $L \longrightarrow K_{1} \oplus P \longrightarrow P_{0} \longrightarrow \Sigma L$ are both triangles in $\xi$. Since $Q$ is $\xi$-SCprojective, by Theorem 2.12, $\xi-\mathcal{S C p d} L=\xi-\mathcal{S} \operatorname{Cpd}(K \oplus P)$. Since $P_{0}$ is $\xi$-SCprojective, by Theorem 2.12 , $\xi$ $\mathcal{S} \operatorname{Cpd} L=\xi-\mathcal{S} \operatorname{Cpd}\left(K_{1} \oplus P\right)$. Thus $\xi-\mathcal{S} \operatorname{Cpd}(K \oplus P)=\xi-\mathcal{S} \operatorname{Cpd}\left(K_{1} \oplus P\right)$. But $K \longrightarrow K \oplus P \longrightarrow P \longrightarrow \Sigma K$ and $K_{1} \longrightarrow K_{1} \oplus P \longrightarrow P \longrightarrow \Sigma K_{1}$ are split triangles and so are in $\xi$, and $P$ is $\xi$-projective, so is $\xi$-SCprojective. By Theorem 2.12 again, then $\xi-\mathcal{S} \mathcal{C p d} K=\xi-\mathcal{S} \mathcal{C p d} K_{1}$. The proof now can be completed by induction.

(ii) $\Rightarrow(\mathrm{i})$. Since $\mathcal{C}$ has enough $\xi$-projectives, there exists a $\xi$-exact complex

$$
0 \longrightarrow B \longrightarrow P_{n-1} \longrightarrow \cdots \longrightarrow P_{0} \longrightarrow A \longrightarrow 0,
$$

where each $P_{i}$ is $\xi$-projective. So by assumption $B$ is $\xi$-SCprojective. This gives the result. 
2.15. Proposition. Assume that $\mathcal{C}$ is a triangulated category with enough $\xi$-projective objects and let $\xi$-SCpd $A \leq 1$ and $\xi x t_{\xi}^{1}(A, P)=0$ for all $P \in \mathcal{P}(\xi)$. Then $A$ is $\xi$-SCprojective.

Proof. Since $\mathcal{C}$ has enough $\xi$-projectives. We have a triangle $K \stackrel{f}{\longrightarrow} P_{0} \stackrel{g}{\longrightarrow} A \stackrel{h}{\longrightarrow} \Sigma K$ in $\xi$, where $P_{0}$ is $\xi$-projective. By proposition $2.14, K$ is $\xi$-SCprojective. Thus we have the following commutative diagram for any $P \in \mathcal{P}(\xi)$ :

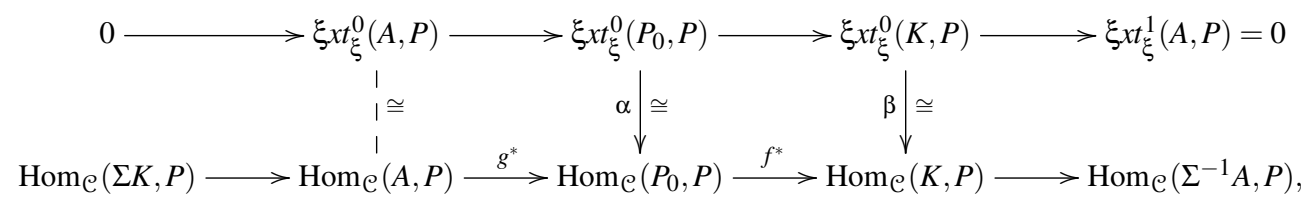

in which the rows are exact. Since the two isomorphisms $\alpha, \beta$ hold by Lemma $2.7, f^{*}$ is epic. So $g^{*}$ is monic. Hence $K \stackrel{f}{\longrightarrow} P_{0} \stackrel{g}{\longrightarrow} A \stackrel{h}{\longrightarrow} \Sigma K$ is $\operatorname{Hom}_{\mathcal{C}}(-, \mathcal{P}(\xi))$ exact. Since $\mathcal{C}$ has enough $\xi$-projectives, we have a triangle $K_{1} \longrightarrow P_{1} \longrightarrow K \longrightarrow \Sigma K_{1}$ in $\xi$ with $P_{1} \xi$-projective. Thus $K_{1}$ is $\xi$-SCprojective by Theorem 2.9. By Proposition 2.6 and Lemma 2.7, there is an exact sequence

$$
0 \longrightarrow \xi x t_{\xi}^{0}(K, P) \longrightarrow \xi x t_{\xi}^{0}\left(P_{1}, P\right) \longrightarrow \xi x t_{\xi}^{0}\left(K_{1}, P\right) \longrightarrow 0 .
$$

This is equivalent to

$$
0 \longrightarrow \operatorname{Hom}_{\mathcal{C}}(K, P) \longrightarrow \operatorname{Hom}_{\mathcal{C}}\left(P_{1}, P\right) \longrightarrow \operatorname{Hom}_{\mathcal{C}}\left(K_{1}, P\right) \longrightarrow 0
$$

is exact. So $K_{1} \longrightarrow P_{1} \longrightarrow K \longrightarrow \Sigma K_{1}$ is also $\operatorname{Hom}_{\mathcal{C}}(-, \mathcal{P}(\xi))$ exact. Proceeding this procedure, we get $\xi$-projective resolution of $A$ satisfying the condition of definition of $\xi$-SCprojective object.

2.16. Theorem. Assume that $\mathcal{C}$ is a triangulated category with enough $\xi$-projective objects and let $A \in \mathcal{C}$ be of finite $\xi$-SCprojective dimension. Then $\xi$-Sepd $A \leq n$ if and only if $\xi x t_{\xi}^{i}(A, Q)=0$ for any $Q \in \widetilde{\mathcal{P}}(\xi)$ and $i>n$.

Proof. Let $\xi$-SCpd $A \leq n$. So there exists a $\xi$-exact complex

$$
0 \longrightarrow P_{n} \longrightarrow P_{n-1} \longrightarrow \cdots \longrightarrow P_{1} \longrightarrow P_{0} \longrightarrow A \longrightarrow 0
$$

with $P_{i} \xi$-SCprojective. But now in view of Lemma 2.7 and using the corresponding triangles, we see that $\xi x t_{\xi}^{i}\left(P_{n}, Q\right) \cong \xi x t_{\xi}^{n+i}(A, Q)=0$ for all $i \geq 1$.

Let $0 \longrightarrow B \longrightarrow P_{n-1} \longrightarrow \cdots \longrightarrow P_{1} \longrightarrow P_{0} \longrightarrow A \longrightarrow 0$ is $\xi$-exact sequence with $P_{i} \xi$ projective. Since $\xi$-Sepd $A<\infty, \xi-\mathcal{S} \mathcal{C p d} B<\infty$. Suppose $\xi$-Sepd $B=m$. Then there exists an $\xi$-exact SCprojective resolution

$$
0 \longrightarrow G_{m} \longrightarrow G_{m-1} \longrightarrow \cdots \longrightarrow G_{0} \longrightarrow B \longrightarrow 0,
$$

with $G_{i} \xi$-SCprojective. Next we show that $B$ is $\xi$-SCprojective. Consider a triangle $G_{m} \longrightarrow$ $G_{m-1} \longrightarrow K_{m-1} \longrightarrow \Sigma G_{m}$ in $\xi$, where $\xi-\mathcal{S} \mathcal{C p d} K_{m-1} \leq 1$. For any $Q \in \mathcal{P}(\xi), \xi x t_{\xi}^{1}\left(K_{m-1}, Q\right) \cong$ $\xi x t_{\xi}^{m}(B, Q) \cong \xi x t_{\xi}^{m+n}(A, Q)=0$. By Proposition $2.15, K_{m-1}$ is $\xi$-SCprojective. Proceeding this procedure, we get $B$ is $\xi$-SCprojective. So $\xi-\mathcal{S} \mathcal{C p d} A \leq n$.

\section{3. $\xi$-SCprojective precover}

3.1. Definition. Let $A$ be an object of $\mathcal{C}$. A morphism $G \longrightarrow A$ where $G$ is $\xi$-SCprojective is called a $\xi$-SCprojective precover of $A$ if it can be completed to an $\operatorname{Hom}_{\mathcal{C}}(\mathcal{S C P}(\xi),-)$-exact triangle $K \longrightarrow G \longrightarrow A \longrightarrow \Sigma K$ in $\xi$.

The following proposition implies that the existence of $\xi$-SCprojective precover.

3.2. Theorem. Let $A$ be an object of $\mathcal{C}$ of finite $\xi$-projective dimension. Then there exists an $\xi$-SCprojective precover. 
Proof. By definition of $\xi$-projective dimension in [3], there exists a triangle $K \stackrel{f}{\longrightarrow} P \stackrel{g}{\longrightarrow} A \stackrel{h}{\longrightarrow}$ $\Sigma K$ with $P \xi$-projective and $\xi$-pd $K<\infty$. For any $\xi$-SCprojective object $Q, \xi x t_{\xi}^{1}(Q, K)=0$ by Lemma 2.7. But $\operatorname{Hom}_{\mathcal{C}}(Q,-)$ is a cohomological functor, then we have the following commutative diagram:

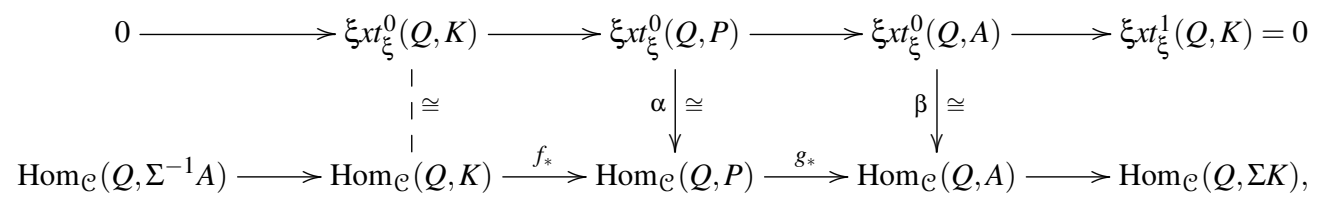

in which the rows are exact. Since the two isomorphisms $\alpha, \beta$ hold by Lemma 2.7, $g_{*}$ is epic. Thus $f_{*}$ is monic. Hence $K \stackrel{f}{\longrightarrow} P \stackrel{g}{\longrightarrow} A \stackrel{h}{\longrightarrow} \Sigma K$ is $\operatorname{Hom}_{\mathcal{C}}(\mathcal{S C P}(\xi),-)$-exact. Then $P \longrightarrow A$ is a $\xi$-Seprojective precover of $A$.

3.3. Proposition. Assume that $K_{1} \stackrel{f_{1}}{\longrightarrow} P_{1} \stackrel{g_{1}}{\longrightarrow} A \stackrel{h_{1}}{\longrightarrow} \Sigma K_{1}$ and $K_{2} \stackrel{f_{2}}{\longrightarrow} P_{2} \stackrel{g_{2}}{\longrightarrow} A \stackrel{h_{2}}{\longrightarrow} \Sigma K_{2}$ are triangles in $\xi$, where $P_{1} \stackrel{g_{1}}{\longrightarrow} A$ and $P_{2} \stackrel{g_{1}}{\longrightarrow} A$ are both $\xi$-SCprojective precovers of $A$. Then $K_{1} \oplus P_{2} \cong K_{2} \oplus P_{1}$.

Proof. According to the base change in Proposition 1.1, we get the following commutative diagram:

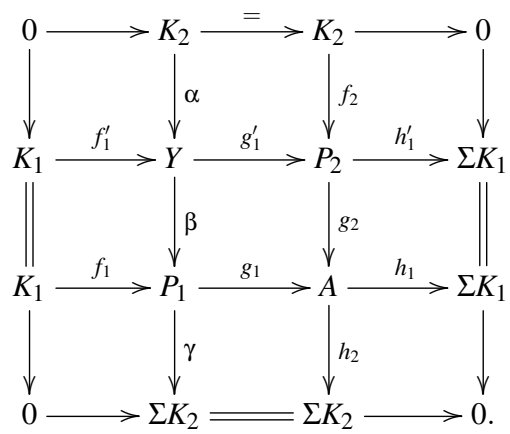

Since $P_{2} \stackrel{g_{2}}{\longrightarrow} A$ is an $\xi$-SCprojective precover of $A$, there is an exact sequence

$$
\operatorname{Hom}_{\mathcal{C}}\left(P_{2}, K_{1}\right) \stackrel{\alpha}{\longrightarrow} \operatorname{Hom}_{\mathcal{C}}\left(P_{2}, P_{1}\right) \longrightarrow \operatorname{Hom}_{\mathcal{C}}\left(P_{2}, A\right) \stackrel{\left(h_{1}\right)_{*}}{\longrightarrow} \operatorname{Hom}_{\mathcal{C}}\left(P_{2}, \Sigma K_{1}\right),
$$

such that $\left(h_{1}\right)_{*} g_{2}=0$, i.e. $h_{1} g_{2}=0$. Thus $h_{1}^{\prime}=0$. Then the second rows is split. Hence $Y \cong K_{1} \oplus P_{2}$. Since $P_{1} \stackrel{g_{1}}{\longrightarrow} A$ is an $\xi$-SCprojective precover of $A$, there is an exact sequence

$$
\operatorname{Hom}_{\mathcal{C}}\left(P_{1}, K_{2}\right) \stackrel{\alpha}{\longrightarrow} \operatorname{Hom}_{\mathcal{C}}\left(P_{1}, P_{2}\right) \longrightarrow \operatorname{Hom}_{\mathcal{C}}\left(P_{1}, A\right) \stackrel{\left(h_{2}\right)_{*}}{\longrightarrow} \operatorname{Hom}_{\mathcal{C}}\left(P_{1}, \Sigma K_{2}\right),
$$

such that $\left(h_{2}\right)_{*} g_{1}=0$, i.e. $h_{2} g_{1}=0$. Thus $\gamma=0$. Then the second column is split. So $Y \cong K_{2} \oplus P_{1}$. Hence $K_{2} \oplus P_{1} \cong K_{1} \oplus P_{2}$.

3.4. Definition. A $\xi$-SCprojective resolution of $A \in \mathcal{C}$ is a $\xi$-exact complex

$$
\mathbf{P}:=\cdots P_{n+1} \stackrel{d^{n+1}}{\longrightarrow} P_{n} \longrightarrow \cdots \longrightarrow P_{1} \longrightarrow P_{0} \longrightarrow A \longrightarrow 0
$$

such that $P_{n} \in \operatorname{SeP}(\xi)$ and for any $n \in \mathbb{N}_{0}$, in the relevant triangle $K_{n} \longrightarrow P_{n} \longrightarrow K_{n-1} \longrightarrow \Sigma K_{n}(n \geq$ 0) $P_{n} \longrightarrow K_{n-1}$ is the $\xi$-SCprojective precover of $K_{n-1}$, in which $K_{-1}=A$. The resolution is said to be of length $n$ if $P_{n} \neq 0$ and $P_{i}=0$ for all $i>n$.

3.5. Definition. Let $\mathbf{P}:=\cdots P_{n+1} \stackrel{d^{n+1}}{\longrightarrow} P_{n} \longrightarrow \cdots \longrightarrow P_{1} \longrightarrow P_{0} \longrightarrow A \longrightarrow 0$ be an $\xi$-SCprojective resolution of $A \in \mathcal{C}$. Then define $\xi_{x} t_{\mathcal{S} \mathcal{C P}(\xi)}^{n}(A, B)$ to be the $n$ th-cohomology of the induced complex $\operatorname{Hom}_{\mathcal{C}}(\mathbf{P}, B)$ for any $B \in \mathcal{C}$. 
Remark. By the comparison theorem the above $\xi$-derived functors are well defined.

3.6. Corollary. Let $0 \longrightarrow P_{n} \longrightarrow P_{n-1} \longrightarrow \cdots \longrightarrow P_{0} \longrightarrow A_{1} \longrightarrow 0$ and $0 \longrightarrow P_{n}^{\prime} \longrightarrow P_{n-1}^{\prime} \longrightarrow$ $\cdots \longrightarrow P_{0}^{\prime} \longrightarrow A_{2} \longrightarrow 0$ be $\xi$-SCprojective resolution of $A_{1}$ and $A_{2}$ respectively. If $A_{1} \cong A_{2}$, then $P_{0} \oplus P_{1}^{\prime} \oplus P_{2} \oplus \cdots \cong P_{0}^{\prime} \oplus P_{1} \oplus P_{2}^{\prime} \oplus \cdots$.

3.7. Proposition. Suppose $A \stackrel{\alpha}{\longrightarrow} B \stackrel{\beta}{\longrightarrow} C \stackrel{\gamma}{\longrightarrow} \Sigma A$ in $\xi$ such that $0 \longrightarrow \operatorname{Hom}_{\mathcal{C}}(P, A) \longrightarrow$ $\operatorname{Hom}_{\mathcal{C}}(P, B) \longrightarrow \operatorname{Hom}_{\mathcal{C}}(P, C) \longrightarrow 0$ is exact for all $P \in \operatorname{SeP}(\xi)$. If $\cdots \longrightarrow P_{1}^{\prime} \longrightarrow P_{0}^{\prime} \stackrel{f_{0}^{\prime}}{\longrightarrow} A \longrightarrow 0$ and $\cdots \longrightarrow P_{1}^{\prime \prime} \longrightarrow P_{0}^{\prime \prime} \stackrel{f_{0}^{\prime \prime}}{\longrightarrow} C \longrightarrow 0$ are $\xi$-SCprojective resolutions of $A$ and $C$ respectively, then there exists a $\xi$-SCprojective resolution of $B$.

Proof. Since $0 \longrightarrow \operatorname{Hom}_{\mathcal{C}}\left(P_{0}^{\prime \prime}, A\right) \longrightarrow \operatorname{Hom}_{\mathcal{C}}\left(P_{0}^{\prime \prime}, B\right) \longrightarrow \operatorname{Hom}_{\mathcal{C}}\left(P_{0}^{\prime \prime}, C\right) \longrightarrow 0$ is exact with $P_{0}^{\prime \prime} \in \operatorname{SeP}(\xi), \gamma f_{0}^{\prime \prime}=0$. Using that $\Sigma$ is an automorphism and a result of Verdier (see [16]), the commutative square on the top left corner below is embedded in a diagram

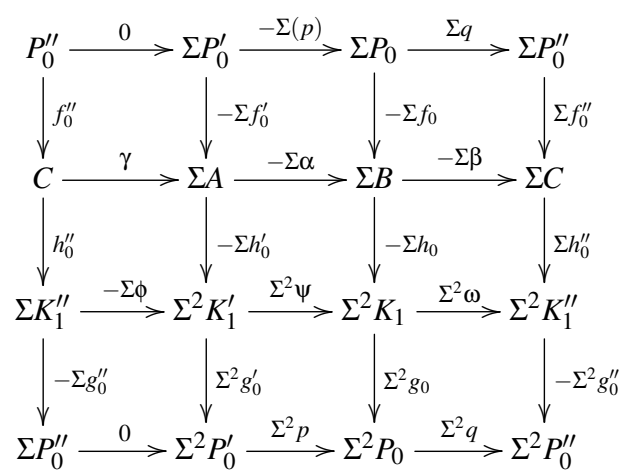

which is commutative except the lower right square which anticommutes and where the rows and columns are triangles. Then we have the following commutative diagram in which the first three vertical and horizontal diagrams are triangles:

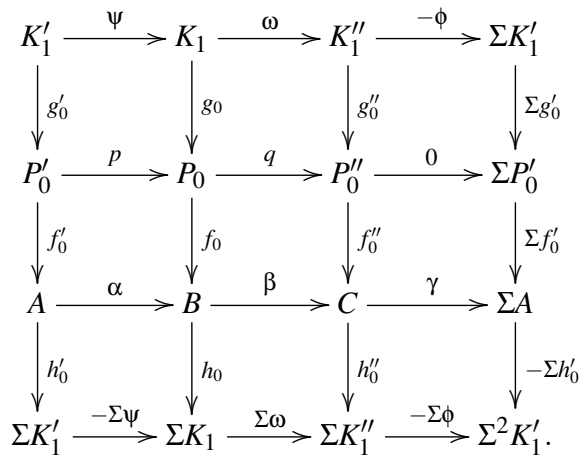

Since the second horizontal triangle is split, we have $P_{0} \in \mathcal{S} \mathcal{P}(\xi)$. Applying the cohomological $\operatorname{Hom}_{\mathcal{C}}(P,-)$ to the above diagram for any $P \in \mathcal{P}(\xi)$, a simple chasing argument shows that the first horizontal triangle and the second vertical triangle are both in $\xi$. Applying to the above diagram the 
cohomological $\operatorname{Hom}_{\mathcal{C}}(Q,-)$ for any $Q \in \operatorname{SeP}(\xi)$, we have the following commutative diagram:

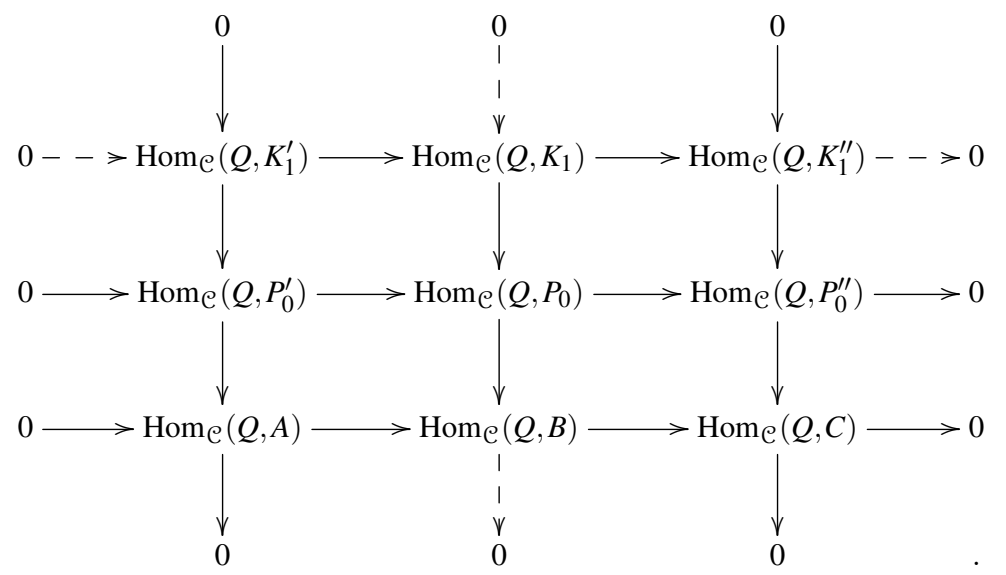

Easily, we get dotted arrows. Then the second row and the second column are both exact. Inductively the above procedure completes the proof.

3.8. Definition. ([3, Definition 4.14]) Let $\mathcal{C}$ be a triangle category and $\mathcal{D}$ is a subcategory of $\mathcal{C}$. $\mathcal{D}$ is called generating subcategory if $\mathcal{D}$ is $\Sigma$-stable and $\operatorname{Hom}_{\mathcal{C}}(\mathcal{D}, A)=0 \Rightarrow A=0$ for any $A \in \mathcal{C}$.

3.9. Theorem. If $\operatorname{SeP}(\xi)$ is a generating subcategory of a triangulated category $\mathcal{C}$, then the following two conditions are equivalent for any $A \in \mathcal{C}$ and $n \geq 0$ :

(i) $\xi x t_{\mathcal{S} \mathcal{P}(\xi)}^{n+1}(A, B)=0$ for any $B \in \mathcal{C}$;

(ii) there exists an $\xi$-SCprojective resolution $0 \longrightarrow P_{n} \longrightarrow P_{n-1} \longrightarrow \cdots \longrightarrow P_{0} \longrightarrow A \longrightarrow 0$.

Proof. (ii) $\Rightarrow$ (i). It is obvious.

(i) $\Rightarrow$ (ii). Let $\cdots \longrightarrow P_{n+2} \stackrel{d_{n+2}}{\longrightarrow} P_{n+1} \stackrel{d_{n+1}}{\longrightarrow} P_{n} \longrightarrow \cdots \longrightarrow P_{1} \longrightarrow P_{0} \longrightarrow A \longrightarrow 0$ be an $\xi$ SCprojective resolution of $A$, where $d_{n}=g_{n-1} f_{n}$ and $P_{n} \stackrel{f_{n}}{\longrightarrow} K_{n}$ is $\xi$-SCprojective precover of $K_{n}$, $\forall n \geq 0$. Since $\xi x t_{\mathcal{S e P}(\xi)}^{n+1}(A, B)=0$ for any $B \in \mathcal{C}$, the complex

$$
\operatorname{Hom}_{\mathcal{C}}\left(P_{n}, K_{n+1}\right) \stackrel{d_{n+1}^{*}}{\longrightarrow} \operatorname{Hom}_{\mathcal{C}}\left(P_{n+1}, K_{n+1}\right) \stackrel{d_{n+2}^{*}}{\longrightarrow} \operatorname{Hom}_{\mathcal{C}}\left(P_{n+2}, K_{n+1}\right)
$$

implies $\operatorname{Im} d_{n+1}^{*}=\operatorname{Ker} d_{n+2}^{*}$. But $f_{n+1} g_{n+1} f_{n+2}=0$, then $f_{n+1} d_{n+2}=0$. That is to say, $d_{n+2}^{*} f_{n+1}=$ 0, i.e. $f_{n+1} \in \operatorname{Ker} d_{n+2}^{*}$. So there exists $\alpha: P_{n} \longrightarrow K_{n+1}$ such that $f_{n+1}=d_{n+1} \alpha$. Applying the functor $\operatorname{Hom}_{\mathcal{C}}(P,-), \forall P \in \mathcal{S} \mathcal{C P}(\xi)$, to the triangle $K_{n+1} \stackrel{g_{n}}{\longrightarrow} P_{n} \stackrel{f_{n}}{\longrightarrow} K_{n} \longrightarrow \Sigma K_{n+1}$, we get the exact sequence

$$
0 \longrightarrow \operatorname{Hom}_{\mathcal{C}}\left(P, K_{n+1}\right) \stackrel{g_{n_{*}}}{\longrightarrow} \operatorname{Hom}_{\mathcal{C}}\left(P, P_{n}\right) \stackrel{f_{n_{*}}}{\longrightarrow} \operatorname{Hom}_{\mathcal{C}}\left(P, K_{n}\right) \longrightarrow 0
$$

Since $\alpha: P_{n} \longrightarrow K_{n+1}$ is $\xi$-SCprojective precover, $\operatorname{Hom}_{\mathcal{C}}\left(P, P_{n}\right) \stackrel{\alpha_{*}}{\longrightarrow} \operatorname{Hom}_{\mathcal{C}}\left(P, K_{n+1}\right)$ is epic. So $\alpha_{*} g_{n_{*}}=1_{\operatorname{Hom}_{\mathcal{C}}\left(P, K_{n+1}\right)}$. Then the above exact sequence is $\operatorname{split} \operatorname{So~}_{\operatorname{Hom}}\left(P, P_{n}\right) \cong \operatorname{Hom}_{\mathcal{C}}\left(P, K_{n} \oplus\right.$ $\left.K_{n+1}\right)$. But $\mathcal{S} \mathcal{C P}(\xi)$ be generating subcategory, then $P_{n} \cong K_{n} \oplus K_{n+1}$. Hence $K_{n}$ is $\xi$-SCprojective. Thus the proof is completed.

Remark. Similar to the way that we define $\xi$-SCprojective objects, one can define $\xi$-SCinjective objects of triangulated category $\mathcal{C}$. The conclusions and their proofs in Sections 2 and 3 dualize perfectly, so all the resluts in these sections have valid analogs in terms of $\xi$-SCinjective objects. 


\section{Conclusions and a future work}

In this paper, we generalize the notion of strongly copure projective modules in category of module to triangulated category, which is called to be $\xi$-strongly copure projective objects. This extends the notions of $\xi$-projective objects and $\xi$-Gorenstein projective objects in triangulated categories. We prove that $\operatorname{SeP}(\xi)$ has a resolving property in Theorem 2.9. We discuss the $\xi$-strongly copure projective dimension and show the relation between $\xi$-SCpdA and $\xi x t_{\xi}^{i}(A,-)$ for any object $A$ of $\mathcal{C}$ in Theorem 2.16. We also introduce the concept of $\xi$-SCprojective precover and investigate the existence in Theorem 3.2, and moreover, characterize the $\xi$-SCprojective resolution of object $A$ by the functor $\xi x t_{\mathcal{S} \mathcal{C P}(\xi)}^{i}(A,-)$ in Theorem 3.9.

Referee of this paper has suggested to study a relative quasi-Frobenius property of the category $\mathcal{C}$ in connection with the results obtained in [8] for module categories and in [14] for locally finitely presented Grothendieck categories. Following Referee's suggestion, we intend to study in future the following problem:

Problem 1. Assume that $\mathcal{C}$ is a triangulated category with enough $\xi$-projective objects as in Section 2. When are the following conditions equivalent?

(i) every $\xi$-SCprojective object in $\mathcal{C}$ is $\xi$-SCinjective;

(ii) every $\xi$-SCinjective object $\mathcal{C}$ is $\xi$-SCprojective; zero),

(iii) every object in $\mathcal{C}$ is $\xi$-SCprojective or $\xi$-SCinjective (that is the global dimension of $\mathcal{C}$ is

Let us recall that the equivalence of these three conditions are proved 40 years ago in [8] for the usual fp-purity in module categories and the equivalence is proved in [14] for the usual fp-purity in any locally finitely presented Grothendieck categories. Moreover, an analogous problem is also discussed in [3].

In the category $R$-Mod of unitary left modules over a ring $R$ with an identity element, the classical equality is

$\sup \left\{\operatorname{pd}_{R} A \mid\right.$ for any left $R$-module $\left.A\right\}=\sup \left\{\operatorname{id}_{R} A \mid\right.$ for any left $R$-module $\left.A\right\}$

established in [13, Theorem 8.14] is extended by D. Bennis and N. Mahdou in [4] to the equality

$$
\sup \left\{\operatorname{Gpd}_{R} A \mid \text { for any left } R \text {-module } A\right\}=\sup \left\{\operatorname{Gid}_{R} A \mid \text { for any left } R \text {-module } A\right\}
$$

where $\operatorname{pd}_{R} A$ (res. $\operatorname{id}_{R} A$ ) means the projective(res. injective) dimension of $A, \operatorname{Gpd}_{R} A\left(\operatorname{res}\right.$. $\operatorname{Gid}_{R} A$ ) means the Gorenstein projective(res. injective) dimension of $A$. Naturally, we also try to find some conditions such that the following conclusion holds in a triangulated category $\mathrm{C}$.

Problem 2. $\sup \{\xi-\mathcal{S} \mathcal{C} \mathrm{d} A \mid$ for any $A \in \mathcal{C}\}=\sup \{\xi-\mathcal{S} \mathcal{C} i d A \mid$ for any $A \in \mathcal{C}\}$.

\section{ACKNOWLEDGEMENTS}

The authors are supported by National Natural Science Foundation of China(No. 11261050).

\section{References}

[1] M. Auslander and M. Bridger, Stable module theory, (Mem.Amer.Math.Soc. 94, 1969.)

[2] J. Asadollahi and Sh. Salarian, Gorenstein objects in triangulated categories, J.Algebra 281, 264-286, 2004.

[3] A. Beligiannis, Relative homological algebra and purity in triangulated categories, J.Algebra 227, 268361, 2000.

[4] D. Bennis and N. Mahdou, Global Gorenstein dimensions, Proc. Amer. Math. Soc. 138, 461-465, 2010.

[5] E.E. Enochs and O.M.G. Jenda, Gorenstein injective and flat dimensions, Math. Japan 44, 261-268, 1996.

[6] E.E. Enochs and O.M.G. Jenda, Copure injective resolutions, flat resolvents and dimensions, Comment. Math. Univ. Carolin 189, 167-193, 1993.

[7] B. Iversen, Octahedra and braids, Bull. Soc. Math. France 114, 197-213, 1986. 
[8] R. Kielpiński and D. Simson, On pure homological diemnsion, Bull. Acad. Polon. Sci., Sér. Sci. Math. 23, 1-6, 1975.

[9] L.X. Mao, Some aspects of strongly P-projective modules and homological dimensions, Comm. Algebra. 41, 19-33, 2013.

[10] J. Miyachi, Localization of triangulated categories and derived categories, J. Algebra 141, 463-483, 1991.

[11] A. Neeman, Triangulated categories, Annals of mathematics studies 148 (Princeton university press, preinceton, NJ, 2001.)

[12] P. Roberts, Homological invariants of modules over a commutative ring, (Lespresses de l'Universitè de Montreal, 1980.)

[13] J. Rotman, An introduction to homological algebra, Academic Press, New York, 2008.

[14] D. Simson, On pure global dimension of locally finitely presented Grothendieck categories, Fund. Math. 96, 91-116, 1977.

[15] J. L Verder, Catégories dérivées:état0,in:SGA 4 $\frac{1}{2}$, Lecture Notes in Math. 569 ( Springer-Verlag, Berlin, 1977$.

[16] C.A. Weibel, An introduction to homological algebra, Cambridge studies in advanced mathematics 38 (Cambridge university press, Cambridge, UK,1994.) 